\title{
Differences in lipid homeostasis and membrane lipid unsaturation confer differential tolerance to low temperatures in two Cycas species
}

Yanling Zheng ${ }^{1} \mathbb{D}$, Yongqiong Yang ${ }^{2}$, Meng Wang ${ }^{1}$, Shijun $\mathrm{Hu}^{1}$, Jianrong $\mathrm{Wu}^{1^{* *}}$ and Zhixiang $\mathrm{Yu}^{2^{*}}$

\begin{abstract}
Background: C. panzhihuaensis is more tolerant to freezing than C. bifida but the mechanisms underlying the different freezing tolerance are unclear. Photosynthesis is one of the most temperature-sensitive processes. Lipids play important roles in membrane structure, signal transduction and energy storage, which are closely related to the stress responses of plants. In this study, the chlorophyll fluorescence parameters and lipid profiles of the two species were characterized to explore the changes in photosynthetic activity and lipid metabolism following lowtemperature exposure and subsequent recovery.
\end{abstract}

Results: Photosynthetic activity significantly decreased in C. bifida with the decrease of temperatures and reached zero after recovery. Photosynthetic activity, however, was little affected in C. panzhihuaensis. The lipid composition of C. bifida was more affected by cold and freezing treatments than C. panzhihuaensis. Compared with the control, the proportions of all the lipid categories recovered to the original level in C. panzhihuaensis, but the proportions of most lipid categories changed significantly in C. bifida after $3 \mathrm{~d}$ of recovery. In particular, the glycerophospholipids and prenol lipids degraded severely during the recovery period of $C$. bifida. Changes in acyl chain length and double bond index (DBI) occurred in more lipid classes immediately after low-temperature exposure in $C$. panzhihuaensis compare with those in C. bifida. DBI of the total main membrane lipids of $C$. panzhihuaensis was significantly higher than that of C. bifida following all temperature treatments.

Conclusions: The results of chlorophyll fluorescence parameters confirmed that the freezing tolerance of $C$. panzhihuaensis was greater than that of C. bifida. The lipid metabolism of the two species had differential responses to low temperatures. The homeostasis and plastic adjustment of lipid metabolism and the higher level of DBI of the main membrane lipids may contribute to the greater tolerance of $C$. panzhihuaensis to low temperatures.

Keywords: Chlorophyll fluorescence parameters, Cold and freezing stress, Cycas, Endangered species, Lipidomics

\footnotetext{
*Correspondence: 565180277@qq.com; flashingzyl@163.com

'Key Laboratory of State Forestry and Grassland Administration for Biodiversity Conservation in Southwest China, Southwest Forestry University, Kunming 650233, Yunnan, China

${ }^{2}$ Administration Bureau of Panzhihua Cycas National Nature Reserve,

Panzhihua 617000, Sichuan, China
}

(c) The Author(s). 2021 Open Access This article is licensed under a Creative Commons Attribution 4.0 International License, which permits use, sharing, adaptation, distribution and reproduction in any medium or format, as long as you give appropriate credit to the original author(s) and the source, provide a link to the Creative Commons licence, and indicate if changes were made. The images or other third party material in this article are included in the article's Creative Commons. licence, unless indicated otherwise in a credit line to the material. If material is not included in the article's Creative Commons licence and your intended use is not permitted by statutory regulation or exceeds the permitted use, you will need to obtain permission directly from the copyright holder. To view a copy of this licence, visit http://creativecommons.org/licenses/by/4.0/. The Creative Commons Public Domain Dedication waiver (http://creativecommons.org/publicdomain/zero/1.0/) applies to the data made available in this article, unless otherwise stated in a credit line to the data. 


\section{Background}

Low temperature is a major threat to plants whose geographical distribution and development are limited. Many plant species in temperate regions can increase their freezing tolerance after exposure to low nonfreezing temperatures, a phenomenon known as cold acclimation [1]. However, some plant species distributed in tropical and subtropical areas may be sensitive to low temperatures above $0{ }^{\circ} \mathrm{C}$ [2]. It has been shown that membrane systems are particularly sensitive to low temperatures [3]. Freezing-induced extracellular ice formation could lead to membrane rupture due to mechanical stress and dehydration of the living cells [4]. Thylakoids possess the most abundant membranes in plant leaves where the light-dependent reactions of photosynthesis occur. Under low temperatures, the balance between light harvesting and light utilization for assimilation may be disrupted [5]. The excessive absorbed energy can lead to oxidative stress by overproducing reactive oxygen species (ROS) [6]. The chloroplast is proven to be the main site of ROS production and ROS attack under stress [7]. Therefore, photosynthesis is extremely sensitive to cold/ freezing stress in plants [8]. However, some plant species have evolved adaptive mechanisms to minimize the negative effects of freezing temperatures [4]. Some molecular, metabolic and physiological characteristics are modulated to enhance plant freezing tolerance $[4,9]$.

Lipids function in membrane structure, signal transduction and energy storage. Lipid composition varies among plant species, tissues and membranes and is affected by the developmental stage and environmental conditions [10, 11]. Lipid metabolism is closely related to plant development and stress response of plants. Membranes are the primary sites of low-temperature injury in plants $[12,13]$. Therefore, membrane properties such as integrity and temperature-compatible fluidity are important for maintaining plant functions and enhancing survival under low temperatures [14]. Glycerophospholipids and saccharolipids are the main membrane lipids. The unsaturation level and acyl chain length of these lipids affect the membrane fluidity [15]. To cope with the adverse effects of low temperature, lipid compositions are adjusted to increase membrane fluidity. The amounts of bilayer-stabilizing lipids such as phosphatidylcholine (PC) and digalactosyldiacylglycerol (DGDG) are also increased [15, 16]. In addition to membrane lipids, glycerolipids are involved in plant tolerance to low temperature due to their functions in intracellular homeostasis and energy balance $[17,18]$. The conversion of diacylglycerol (DAG) to triacylglycerol (TAG) contributes to freezing tolerance of some plant species [3].

Cycads are considered to be living fossils due to their primitive features. Cycad study has evolutionary and adaptive implications in the context of global environmental changes. More than $60 \%$ of the known cycad species are in danger of extinction [19]. Cycas is the oldest genus of cycads and these species are restricted to the tropical and subtropical areas of Asia, Eastern Africa and Madagascar islands and the Australia Pacific islands [20, 21]. More than 20 species of Cycas occur in China, and all of them have been proposed as first-ranked plants for national protection [22]. Cycas species are considered to be sensitive to low temperature, but there are few studies on the adaptation of these species to cold and freezing temperatures.

C. bifida is one of the rarest cycads in China, and the distribution of this species is restricted to areas of Yunnan and Guangxi provinces. C. panzhihuaensis is endemic to the dry-hot valleys of the Jinsha River in southwest China. Its natural distribution is in the northernmost limit areas and at the highest altitude among the Cycas species. The natural distributions of $C$. bifida and $C$. panzhihuaensis in China are restricted to the subtropical zone. Previous research has showed that the freezing tolerance of $C$. panzhihuaensis is greater than that of C. bifida [23]. However, the responses of these species to low temperatures and subsequent recovery conditions are unclear. The goal of this study was to dissect the effects of cold, freezing and subsequent recovery on photosynthetic activities and lipid metabolism of $C$. bifida and $C$. multipinnata. The results can increase understanding of the freezing sensitivity and distribution of the two species.

\section{Results}

\section{Changes in chlorophyll fluorescence parameters}

Chlorophyll fluorescence is a non-invasive and highly sensitive probe used for monitoring the effects of environmental stresses on photosynthesis. Fv/Fm, Y(II) and rETR of $C$. bifida decreased significantly with the decrease of temperature, reaching the lowest level (near zero) after $3 \mathrm{~d}$ of recovery (Table 1). However, $\mathrm{Y}(\mathrm{NO})$ of C. bifida displayed an opposite trend, increasing by $323.21 \%$ for the recovered seedlings compared to the control. Y(NPQ) of C. bifida decreased significantly in seedlings subjected to freezing and recovery treatments, by 75.87 and $83.49 \%$, respectively. $\mathrm{qP}$ and $\mathrm{qN}$ of $C$. bifida decreased significantly in seedlings subjected to freezing treatment, then $\mathrm{qP}$ increased to the control level and $\mathrm{qN}$ increased significantly following recovery. These data suggest that cold diminished the photosynthetic activity and freezing severely damaged the photosynthetic apparatus of $C$. bifida. Fv/Fm of C. panzhihuaensis decreased significantly in seedlings subjected to cold, freezing and recovery treatments, by 4.96, 5.54 and 5.07\%, respectively. $\mathrm{Y}(\mathrm{NO})$ increased significantly but $\mathrm{qN}$ decreased significantly for seedlings subjected to freezing treatment. Except for $\mathrm{Fv} / \mathrm{Fm}, \mathrm{Y}(\mathrm{NO})$ and $\mathrm{qN}$, all other 
Table 1 Effects of low temperature treatments on chlorophyll fluorescence parameters of C. bifida and C. panzhihuaensis

\begin{tabular}{|c|c|c|c|c|c|}
\hline \multirow[t]{2}{*}{ Parameters } & \multirow[t]{2}{*}{ Species } & \multicolumn{4}{|l|}{ Treatment } \\
\hline & & Control & Cold & Freezing & Recovery \\
\hline \multirow[t]{2}{*}{$\mathrm{Fv} / \mathrm{Fm}$} & C. bifida & $0.826 \pm 0.006 a$ & $0.782 \pm 0.015 b$ & $0.717 \pm 0.046 c$ & $0.001 \pm 0.002 d$ \\
\hline & C. panzhihuaensis & $0.857 \pm 0.010 a^{*}$ & $0.824 \pm 0.004 b^{*}$ & $0.819 \pm 0.007 b^{*}$ & $0.823 \pm 0.009 b^{*}$ \\
\hline \multirow[t]{2}{*}{$Y(I I)$} & C. bifida & $0.462 \pm 0.068 a$ & $0.325 \pm 0.052 b$ & $0.152 \pm 0.040 c$ & $0 \pm 0 d$ \\
\hline & C. panzhihuaensis & $0.465 \pm 0.050 a$ & $0.421 \pm 0.044 a b^{*}$ & $0.427 \pm 0.018 a b^{*}$ & $0.383 \pm 0.026 b^{*}$ \\
\hline \multirow[t]{2}{*}{ Y(NPQ) } & C. bifida & $0.315 \pm 0.084 a$ & $0.321 \pm 0.045 a$ & $0.076 \pm 0.057 b$ & $0.052 \pm 0.005 b$ \\
\hline & C. panzhihuaensis & $0.260 \pm 0.046 b$ & $0.288 \pm 0.034 a b$ & $0.226 \pm 0.030 b^{*}$ & $0.313 \pm 0.034 a^{*}$ \\
\hline \multirow[t]{2}{*}{$\mathrm{Y}(\mathrm{NO})$} & C. bifida & $0.224 \pm 0.016 d$ & $0.354 \pm 0.014 c$ & $0.776 \pm 0.104 b$ & $0.948 \pm 0.005 a$ \\
\hline & C. panzhihuaensis & $0.275 \pm 0.016 b^{*}$ & $0.291 \pm 0.039 b^{*}$ & $0.348 \pm 0.023 a^{*}$ & $0.304 \pm 0.013 b^{*}$ \\
\hline \multirow[t]{2}{*}{ qp } & C. bifida & $0.744 \pm 0.044 a$ & $0.533 \pm 0.061 \mathrm{ab}$ & $0.241 \pm 0.054 b$ & $0.8 \pm 0.0447 a$ \\
\hline & C. panzhihuaensis & $0.635 \pm 0.048 a^{*}$ & $0.644 \pm 0.070 a^{*}$ & $0.634 \pm 0.019 a^{*}$ & $0.588 \pm 0.028 a^{*}$ \\
\hline \multirow[t]{2}{*}{$q N$} & C. bifida & $0.682 \pm 0.106 b$ & $0.596 \pm 0.043 b$ & $0.134 \pm 0.100 c$ & $0.987 \pm 0.029 a$ \\
\hline & C. panzhihuaensis & $0.561 \pm 0.050 a^{*}$ & $0.602 \pm 0.054 a$ & $0.490 \pm 0.051 b^{*}$ & $0.612 \pm 0.043 a^{*}$ \\
\hline \multirow[t]{2}{*}{ rETR } & C. bifida & $120.4 \pm 17.501 a$ & $83.6 \pm 13.390 b$ & $38 \pm 9.925 c$ & $0 \pm 0 d$ \\
\hline & C. panzhihuaensis & $117.4 \pm 12.740 \mathrm{a}$ & $108.6 \pm 11.127 a^{*}$ & $108.6 \pm 4.393 a^{*}$ & $94.8 \pm 6.686 b^{*}$ \\
\hline
\end{tabular}

Different letters in the same row indicate significant differences between treatments within species and * indicates significant difference between species within treatment $(P<0.05)$. Data are mean \pm standard deviation $(n=4)$

FV/Fm The maximum quantum yield of photosystem II (PSII), qP Photochemical quenching coefficient, $q N$ Non-photochemical quenching coefficient, $r E T R$ Relative electron transport rate, $Y(I I)$ Effective quantum yield of PS II, Y(NO) Non-regulated non-photochemical energy loss in PS II, Y(NPQ) Regulated non-photochemical energy loss in PS II

Table 2 The proportion of each lipid category in C. bifida and C. panzhihuaensis treated at low temperatures

\begin{tabular}{|c|c|c|c|c|c|}
\hline \multirow[t]{3}{*}{ Lipid category } & \multirow[t]{3}{*}{ Species } & \multicolumn{4}{|l|}{ Treatment } \\
\hline & & Control & Cold & Freezing & Recovery \\
\hline & & \multicolumn{4}{|c|}{ Lipid proportion (\%) } \\
\hline \multirow[t]{2}{*}{ Neutral glycerolipids } & C. bifida & $15.50 \pm 6.63 b$ & $7.11 \pm 1.78 \mathrm{c}$ & $8.76 \pm 2.04 c$ & $23.86 \pm 1.16 a$ \\
\hline & C. panzhihuaensis & $16.72 \pm 6.93 a$ & $9.35 \pm 1.89 b$ & $17.81 \pm 4.63 a^{*}$ & $15.12 \pm 0.80 a^{*}$ \\
\hline \multirow[t]{2}{*}{ Glycerophospholipids } & C. bifida & $31.09 \pm 5.19 b$ & $40.77 \pm 3.67 a$ & $35.90 \pm 6.46 a b$ & $21.87 \pm 2.38 \mathrm{c}$ \\
\hline & C. panzhihuaensis & $21.99 \pm 1.03 b^{*}$ & $20.58 \pm 1.84 b^{*}$ & $24.58 \pm 1.64 a^{*}$ & $21.11 \pm 1.32 b$ \\
\hline \multirow[t]{2}{*}{ Saccharolipids } & C. bifida & $44.69 \pm 4.48 a$ & $44.02 \pm 5.24 a$ & $46.47 \pm 4.19 a$ & $41.47 \pm 2.81 \mathrm{a}$ \\
\hline & C. panzhihuaensis & $54.86 \pm 7.14 b^{*}$ & $65.25 \pm 1.91 a^{*}$ & $50.55 \pm 5.31 b$ & $55.87 \pm 2.26 b^{*}$ \\
\hline \multirow[t]{2}{*}{ Lysophospholipids } & C. bifida & $1.92 \pm 0.94 a$ & $3.06 \pm 0.92 a$ & $3.06 \pm 0.68 \mathrm{a}$ & $2.15 \pm 0.28 a$ \\
\hline & C. panzhihuaensis & $0.51 \pm 0.28 a^{*}$ & $0.59 \pm 0.14 a^{*}$ & $0.43 \pm 0.29 a^{*}$ & $0.76 \pm 0.15 a^{*}$ \\
\hline \multirow[t]{2}{*}{ Sphingolipids } & C. bifida & $2.89 \pm 0.79 b$ & $2.25 \pm 1.05 b$ & $2.27 \pm 0.45 b$ & $6.03 \pm 1.19 a$ \\
\hline & C. panzhihuaensis & $2.17 \pm 0.50 \mathrm{a}$ & $1.60 \pm 0.24 a$ & $2.25 \pm 0.59 a$ & $2.27 \pm 0.61 a^{*}$ \\
\hline \multirow[t]{2}{*}{ Sterol lipids } & C. bifida & $1.51 \pm 0.39 b$ & $1.30 \pm 0.39 b$ & $1.85 \pm 0.22 b$ & $3.27 \pm 0.73 a$ \\
\hline & C. panzhihuaensis & $1.48 \pm 0.52 \mathrm{a}$ & $1.29 \pm 0.75 a$ & $1.72 \pm 0.47 a$ & $2.30 \pm 0.43 a^{*}$ \\
\hline \multirow[t]{2}{*}{ Prenol lipids } & C. bifida & $1.97 \pm 0.64 a$ & $1.44 \pm 0.34 a$ & $1.53 \pm 0.25 \mathrm{a}$ & $0.81 \pm 0.18 b$ \\
\hline & C. panzhihuaensis & $2.08 \pm 0.45 a$ & $1.17 \pm 0.35 b$ & $2.17 \pm 0.53 a^{*}$ & $2.05 \pm 0.18 a^{*}$ \\
\hline \multirow[t]{2}{*}{ Fatty acyls } & C. bifida & $0.40 \pm 0.10 \mathrm{a}$ & $0.04 \pm 0.02 b$ & $0.17 \pm 0.04 b$ & $0.55 \pm 0.24 a$ \\
\hline & C. panzhihuaensis & $0.19 \pm 0.08 b^{*}$ & $0.17 \pm 0.03 b^{*}$ & $0.51 \pm 0.20 a^{*}$ & $0.15 \pm 0.04 b^{*}$ \\
\hline
\end{tabular}


parameters remained unchanged following lowtemperature treatments. Both Y(II) and rETR of C. panzhihuaensis decreased significantly but $\mathrm{Y}(\mathrm{NPQ})$ increased significantly for recovered seedlings. Fv/Fm, Y(II) and rETR of C. panzhihuaensis were significantly higher than those of $C$. bifida after the various treatments. These results demonstrate that the photosynthesis of C. panzhihuaensis was only slightly affected by cold, and the photosynthetic apparatus was damaged by freezing to a lesser extent than that of C. bifida.

\section{Lipids profiling in the leaves of C. bifida and C. panzhihuaensis}

A total of 26 lipid classes including 613 lipid species were identified from the leaves of $C$. bifida and $C$. panzhihuaensis (Table S1). The lipids contained two neutral glycerolipid classes, eight classes of glycerophospholipids (excluding lysophospholipids here), four of lysophospholipids, four of saccharolipids, four of sphingolipids, two of sterol lipids, one of prenol lipids (coenzyme Q) and one of fatty acyls (wax esters). The total lipid content of C. bifida was not significantly different from that of C. panzhihuaensis. Neutral glycerolipids, glycerophospholipids and saccharolipids were the main lipid categories for both the species, accounting for more than $90 \%$ of the total lipids (Table 2). The absolute content of neutral glycerolipids, glycerophospholipids and saccharolipids did not differ but the proportions of glycerophospholipids and saccharolipids differed significantly between the two species (Fig. 1; Table 2). The ratio of saccharolipids to phospholipids was significantly higher in C. panzhihuaensis (Table S2). Phosphatidic acid (PA), PC, phosphatidylethanolamine (PE), phosphatidylglycerol (PG) and phosphatidylinositol (PI) were the main glycerophospholipids. Monogalactosyldiacylglycerol (MGDG), DGDG and sulphoquinovosyldiacylglycerol (SQDG) were the main saccharolipids (Table 3).

\section{Changes in the composition of the lipid categories}

The content of total lipids, glycerophospholipids, lysophospholipids, saccharolipids, prenol lipids and sterol lipids in C. bifida increased after the cold and freezing

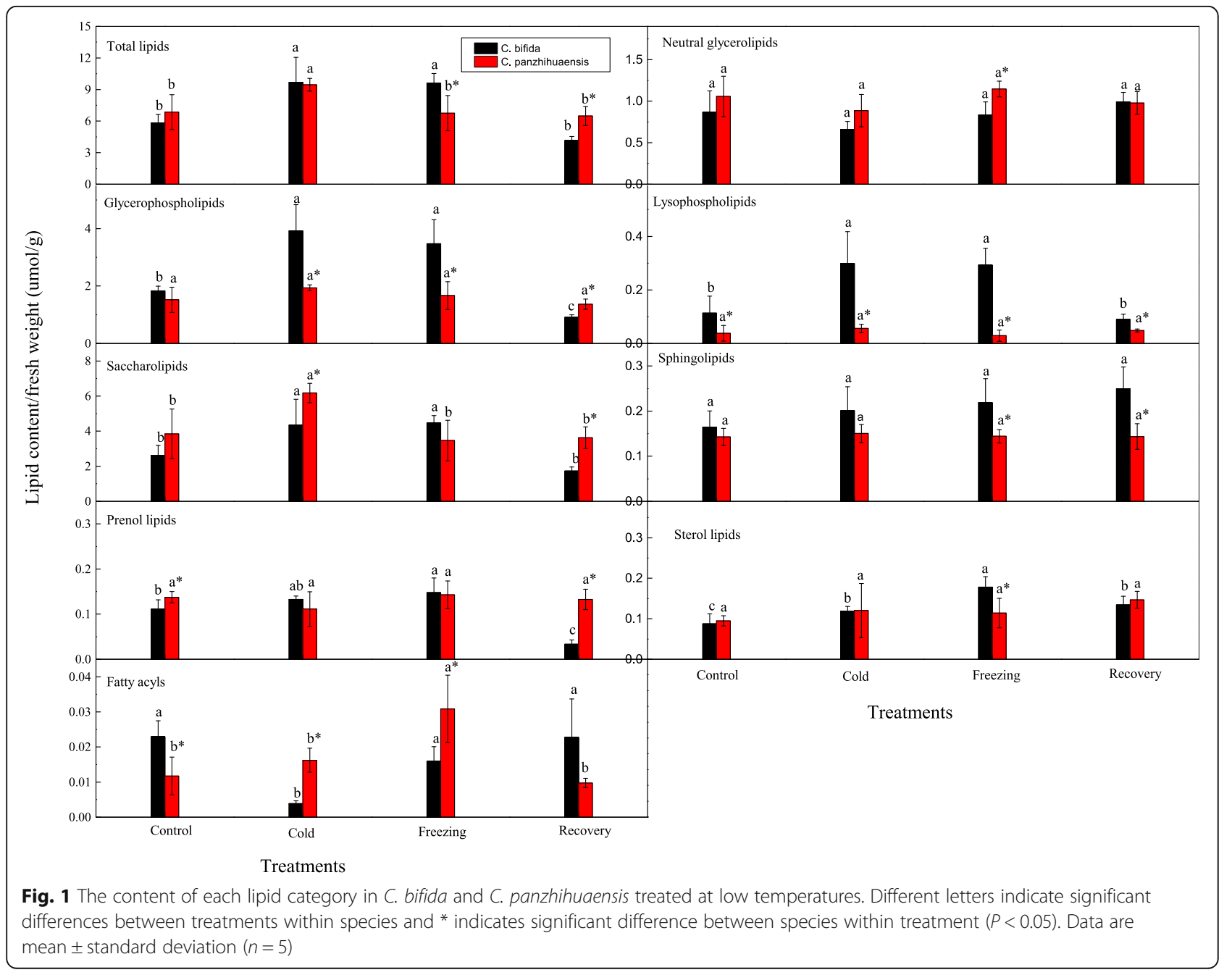


Table 3 The content of each main membrane lipid class in C. bifida and C. panzhihuaensis treated at low temperatures

\begin{tabular}{|c|c|c|c|c|c|}
\hline \multirow[t]{4}{*}{ Lipid class } & \multirow[t]{4}{*}{ Species } & \multicolumn{4}{|l|}{ Treatment } \\
\hline & & Control & Cold & Freezing & Recovery \\
\hline & & \multicolumn{4}{|c|}{ Lipid content $(\mu \mathrm{mol} / \mathrm{g} \mathrm{FW})$} \\
\hline & & Control & Cold & Freezing & Recovery \\
\hline \multirow[t]{2}{*}{ PA } & C. bifida & $0.74 \pm 0.41 b$ & $1.81 \pm 0.59 a$ & $1.27 \pm 0.30 \mathrm{ab}$ & $0.26 \pm 0.06 b$ \\
\hline & C. panzhihuaensis & $0.30 \pm 0.16 a$ & $0.42 \pm 0.06 a^{*}$ & $0.36 \pm 0.05 a^{*}$ & $0.35 \pm 0.07 a$ \\
\hline \multirow[t]{2}{*}{ PC } & C. bifida & $0.21 \pm 0.09 b$ & $0.46 \pm 0.17 a$ & $0.42 \pm 0.08 a$ & $0.13 \pm 0.02 b$ \\
\hline & C. panzhihuaensis & $0.26 \pm 0.15 b$ & $0.41 \pm 0.01 a$ & $0.15 \pm 0.02 b^{*}$ & $0.22 \pm 0.12 b$ \\
\hline \multirow[t]{2}{*}{$P E$} & C. bifida & $0.10 \pm 0.01 c$ & $0.22 \pm 0.04 a$ & $0.17 \pm 0.02 b$ & $0.06 \pm 0.00 d$ \\
\hline & C. panzhihuaensis & $0.12 \pm 0.06 b$ & $0.19 \pm 0.04 a$ & $0.09 \pm 0.02 b^{*}$ & $0.10 \pm 0.04 b$ \\
\hline \multirow[t]{2}{*}{ PG } & C. bifida & $0.27 \pm 0.09 b$ & $0.52 \pm 0.17 a$ & $0.49 \pm 0.05 a$ & $0.18 \pm 0.02 b$ \\
\hline & C. panzhihuaensis & $0.32 \pm 0.04 a$ & $0.28 \pm 0.04 a b^{*}$ & $0.19 \pm 0.03 c^{*}$ & $0.25 \pm 0.02 b^{*}$ \\
\hline \multirow[t]{2}{*}{$\mathrm{Pl}$} & C. bifida & $0.45 \pm 0.15 b$ & $0.85 \pm 0.21 a b$ & $1.06 \pm 0.04 a$ & $0.23 \pm 0.05 b$ \\
\hline & C. panzhihuaensis & $0.47 \pm 0.10 b$ & $0.57 \pm 0.08 a b^{*}$ & $0.78 \pm 0.30 a$ & $0.37 \pm 0.08 b^{*}$ \\
\hline \multirow[t]{2}{*}{ PS } & C. bifida & $0.03 \pm 0.01 b$ & $0.04 \pm 0.01 a$ & $0.04 \pm 0.00 \mathrm{ab}$ & $0.02 \pm 0.00 c$ \\
\hline & C. panzhihuaensis & $0.03 \pm 0.01 a$ & $0.04 \pm 0.00 a$ & $0.04 \pm 0.01 a$ & $0.04 \pm 0.00 a^{*}$ \\
\hline \multirow[t]{2}{*}{ MGMG } & C. bifida & $0.04 \pm 0.01 b$ & $0.07 \pm 0.02 \mathrm{a}$ & $0.07 \pm 0.02 a$ & $0.04 \pm 0.01 b$ \\
\hline & C. panzhihuaensis & $0.08 \pm 0.05 b$ & $0.17 \pm 0.05 a^{*}$ & $0.07 \pm 0.02 b$ & $0.08 \pm 0.02 b^{*}$ \\
\hline \multirow[t]{2}{*}{ MGDG } & C. bifida & $0.50 \pm 0.10 b$ & $1.15 \pm 0.57 a$ & $1.03 \pm 0.22 a$ & $0.55 \pm 0.10 b$ \\
\hline & C. panzhihuaensis & $1.25 \pm 0.76 b$ & $2.64 \pm 0.35 a^{*}$ & $0.65 \pm 0.21 b^{*}$ & $1.02 \pm 0.46 b$ \\
\hline \multirow[t]{2}{*}{ DGDG } & C. bifida & $0.56 \pm 0.07 c$ & $1.13 \pm 0.23 b$ & $1.36 \pm 0.11 a$ & $0.09 \pm 0.01 d$ \\
\hline & C. panzhihuaensis & $0.80 \pm 0.24 b$ & $1.39 \pm 0.17 a$ & $0.84 \pm 0.45 b$ & $0.84 \pm 0.41 b^{*}$ \\
\hline \multirow[t]{2}{*}{ SQDG } & C. bifida & $1.53 \pm 0.36 b$ & $1.99 \pm 0.36 a$ & $2.00 \pm 0.20 a$ & $1.04 \pm 0.13 c$ \\
\hline & C. panzhihuaensis & $1.72 \pm 0.39 a$ & $1.98 \pm 0.17 a$ & $1.91 \pm 0.50 \mathrm{a}$ & $1.69 \pm 0.41 a^{*}$ \\
\hline \multirow[t]{2}{*}{ Total } & C. bifida & $4.46 \pm 0.99 b$ & $8.25 \pm 2.34 a$ & $7.94 \pm 0.90 a$ & $2.63 \pm 0.28 b$ \\
\hline & C. panzhihuaensis & $5.37 \pm 1.86 b$ & $8.12 \pm 0.54 a$ & $5.14 \pm 1.61 b^{*}$ & $5.00 \pm 0.76 b^{*}$ \\
\hline
\end{tabular}

Different letters in the same row indicate significant differences between treatments within species and * indicates significant difference between species within treatment $(P<0.05)$. Data are mean \pm standard deviation $(n=5)$

$D G D G$ Digalactosyldiacylglycerol, MGDG Monogalactosyldiacylglycerol, MGMG monogalactosylmonoacylglycerol, $P A$ Phosphatidic acid, $P C$ Phosphatidylcholine, $P E$ Phosphatidylethanolamine, PG Phosphatidylglycerol, PI Phosphatidylinositol, PS Phosphatidylserine, SQDG Sulphoquinovosyldiacylglycerol

treatments. However, glycerophospholipids and prenol lipids decreased significantly and sterol lipids increased significantly after $3 \mathrm{~d}$ of recovery compared to those of the control (Fig. 1). The content of fatty acyls decreased significantly after cold treatment but recovered to the original level after freezing and recovery treatments. The ratio of saccharolipids to glycerophospholipids of $C$. bifida increased significantly after recovery treatments, which was mainly due to the severe degradation of the glycerophospholipids (Table S2). This suggests that the glycerophospholipids of $C$. bifida were more easily affected by freezing temperature than the saccharolipids. Compared to the control, the relative contents of all the lipid categories except saccharolipids, lysophospholipids and fatty acyls in C. bifida changed significantly after recovery (Table 2). This shows that the lipid metabolic balances of $C$. bifida were disturbed by low temperatures. For C. panzhihuaensis, the content of total lipids and saccharolipids increased significantly after cold treatment, and that of fatty acyls increased significantly after freezing treatment (Fig. 1). The ratio of saccharolipids to glycerophospholipids of C. panzhihuaensis increased significantly after cold treatment which was mainly due to the accumulation of saccharolipids (Table S2). Although the relative contents of neutral glycerolipids, glycerophospholipids, saccharolipids, prenol lipids and fatty acyls changed significantly following cold or freezing treatment, they all returned to the control level after recovery (Table 2). This suggests that the lipid metabolism of $C$. panzhihuaensis could positively respond to low-temperature stress and then recover to the normal state. It can also be concluded that freezing and subsequent thawing did not disturb the lipid metabolic system.

In a comparison of the two species, the contents of glycerophospholipids and lysophospholipids were significantly lower and those of saccharolipids and fatty acyls were significantly higher in C. panzhihuaensis after cold 
treatment. The contents of total lipids, glycerophospholipids, lysophospholipids, sphingolipids and sterol lipids were significantly lower and those of neutral glycerolipids and fatty acyls were significantly higher in C. panzhihuaensis following freezing treatment. After recovery treatment, the contents of total lipids, glycerophospholipids, saccharolipids and prenol lipids were significantly higher and those of lysophospholipids and sphingolipids were significantly lower in C. panzhihuaensis.

\section{Changes in the composition of main lipid classes and} lipid species of glycerophospholipids and saccharolipids Phosphatidylinositol phosphate (PIP) and cardiolipin (CL) were also detected, but their levels were extremely low (Table S3). Therefore, these two lipid classes were not analyzed separately but included in the analysis of total glycerophospholipids. PA, PC, PE, PG, PI and PS contents of $C$. bifida increased significantly after cold and/or freezing treatments (Table 3). However, PA, PC, PG and PI contents recovered to the original level and PE and PS contents decreased significantly after $3 \mathrm{~d}$ of recovery. Compared to the control, PC and PE levels in
C. panzhihuaensis increased significantly after cold treatment (Table 3). PG content decreased significantly but PI content increased significantly after freezing treatment. After recovery, PG content increased significantly compared to that of the freezing-treated seedlings but did not reach the original level. PA, PG and PI levels in C. panzhihuaensis were significantly lower than levels in C. bifida after cold treatment. PA, PC, PE and PG contents of $C$. panzhihuaensis were significantly lower than those of $C$. bifida after freezing treatment. After recovery, PG, PI and PS contents of C. panzhihuaensis were significantly higher than those of $C$. bifida. Among the main lipid species of the several lipid classes, there were significantly different responses of the two species to various treatments in all the PA species except 32:0, 34: 1, 34:2, 34:3 and 43:2; all the PC species except 35:3, 35: 4, 37:3, 37:4 and 37:5; all the PE species except 36:4 and 47:4; all the PG species except 30:2, 34:5, 36:3, 36:4, 44:1 and 46:1; PI species 33:2, 34:1, 36:3, 49:3, 50:2, 50:3 and 51:4; PS species 33:0, 39:4 and 40:8 (Fig. 2).

Compared to those of the control, the content of each class of saccharolipids in C. bifida increased significantly

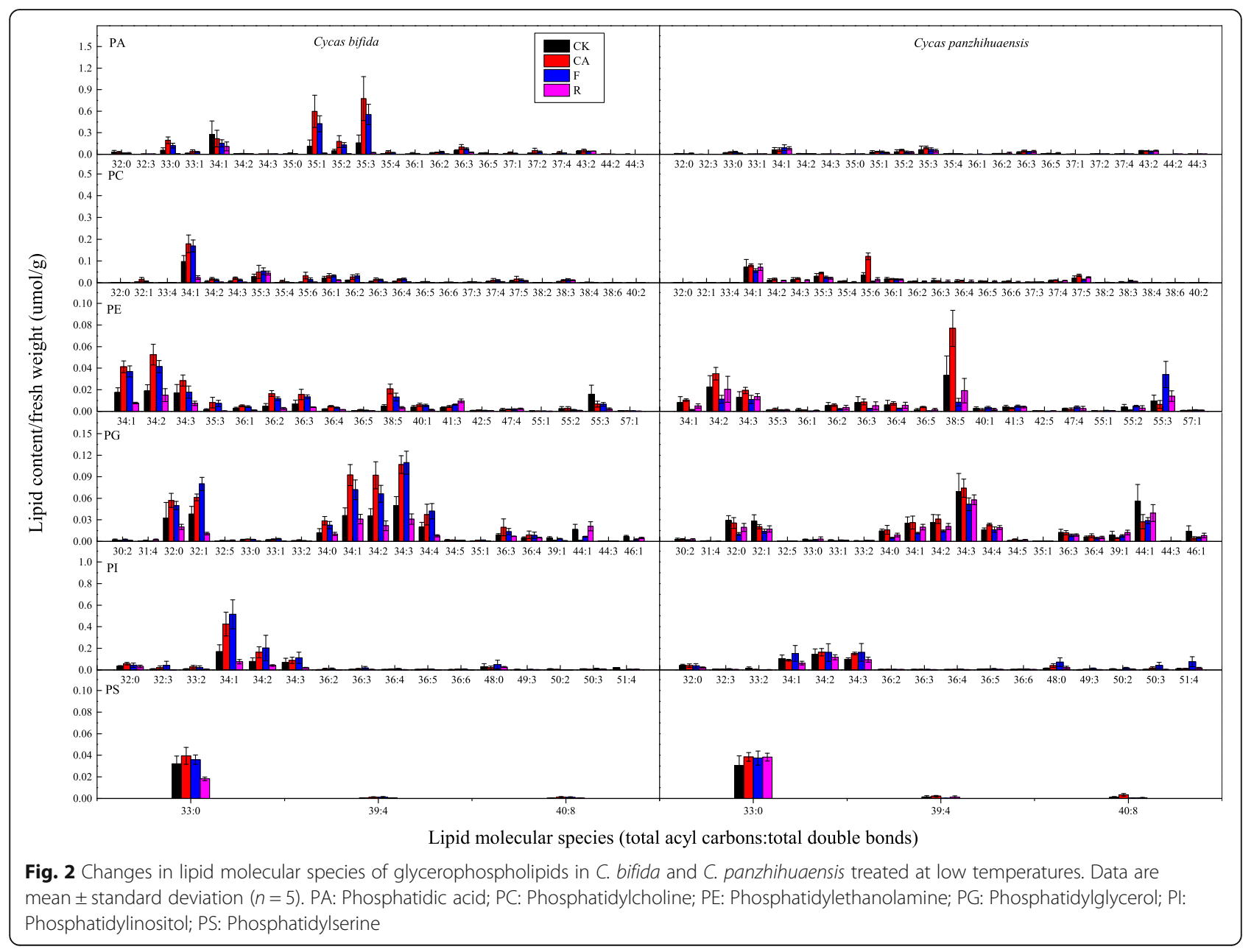


after cold and freezing treatments (Table 3). Monogalactosylmonoacylglycerol (MGMG) and MGDG then returned to the original level but DGDG and SQDG decreased, by 83.93 and $32.03 \%$ respectively, after $3 \mathrm{~d}$ of recovery compared to the control. These results show that the equilibrium of saccharolipid metabolism of C. bifida was disrupted due to the degradation of DGDG and SQDG at the recovery stage. For C. panzhihuaensis, SQDG content did not vary among the control and different treatments (Table 3). MGMG, MGDG and DGDG only responded to cold treatment, increasing by 112.5 , 111.2 and $73.75 \%$, respectively. Compared to those of $C$. bifida, both MGMG and MGDG contents of C. panzhihuaensis were significantly higher in the cold-treated seedlings; MGDG content was significantly lower for the freezing-treated seedlings; MGMG, DGDG and SQDG content were significantly higher for the recovered seedlings. Among the main lipid species of the several lipid classes, there were significantly different responses of the two species to various treatments in MGMG species 18:3; MGDG species 34:2, 34:3, 34:4, 34:5, 34:6, 35:3, 36 : 2 , 36:3, 36:4, 36:6 and 38:6; all the DGDG species except
31:3, 35:3, 36:5, 40:0 and 42:1; SQDG species 36:6, 38:7, 38:9, 41:8 and 44:8 (Fig. 3).

\section{Changes in the $A C L$ and $D B I$ of glycerophospholipids and saccharolipids}

The ACL of total glycerophospholipids, PE and PI of $C$. bifida was not affected by the treatments (Table 4). The ACL of PA and PC was not affected by low temperature but increased significantly after recovery. The ACL of PG decreased significantly after low-temperature treatments but then increased to the control level after recovery. The ACL of PS increased significantly after lowtemperature and recovery treatments. C. panzhihuaensis had significantly different responses to various treatments compared to C. bifida in the ACL of PC, PE, PI and PS (Table 4). The ACL of PA and PE decreased and that of PS increased significantly after cold treatments. The ACL increased significantly for PC, PE, PI and total glycerophospholipids and decreased for PA and PS after freezing treatments. Compared to those in C. bifida, the ACL of most glycerophospholipid classes in C. panzhihauensis was higher following cold or/and freezing

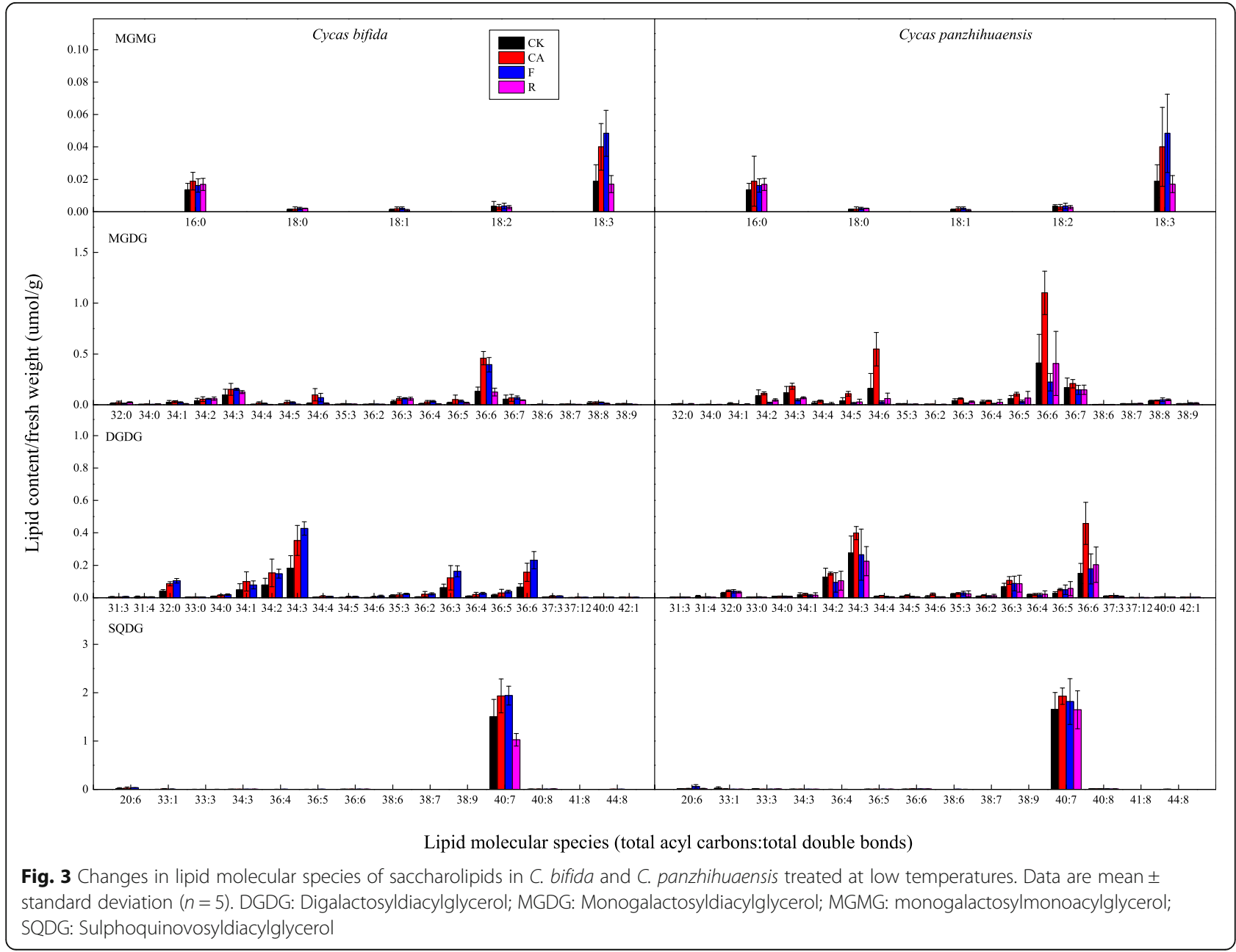


Table 4 Acyl chain length (ACL) of each main membrane lipid class in C. bifida and C. panzhihuaensis treated at low temperatures

\begin{tabular}{|c|c|c|c|c|c|}
\hline \multirow[t]{3}{*}{ Lipid class } & \multirow[t]{3}{*}{ Species } & \multirow{3}{*}{$\begin{array}{l}\text { Control } \\
\mathrm{ACL}\end{array}$} & \multicolumn{2}{|l|}{ Treatment } & \multirow{3}{*}{ Recovery } \\
\hline & & & Cold & Freezing & \\
\hline & & & & & \\
\hline \multirow[t]{2}{*}{ PA } & C. bifida & $35.26 \pm 0.67 b$ & $35.03 \pm 0.10 b$ & $35.08 \pm 0.08 b$ & $35.91 \pm 0.51 a$ \\
\hline & C. panzhihuaensis & $36.36 \pm 0.62 a^{*}$ & $35.73 \pm 0.16 b^{*}$ & $35.70 \pm 0.21 b^{*}$ & $36.00 \pm 0.32 \mathrm{ab}$ \\
\hline \multirow[t]{2}{*}{ PC } & C. bifida & $34.99 \pm 0.27 b$ & $34.89 \pm 0.11 b$ & $34.99 \pm 0.06 b$ & $35.50 \pm 0.09 a$ \\
\hline & C. panzhihuaensis & $35.13 \pm 0.07 b$ & $35.11 \pm 0.04 b^{*}$ & $35.33 \pm 0.10 a^{*}$ & $35.12 \pm 0.17 b^{*}$ \\
\hline \multirow[t]{2}{*}{ PE } & C. bifida & $39.20 \pm 4.61 a$ & $36.26 \pm 0.37 a$ & $36.37 \pm 0.16 a$ & $37.61 \pm 0.98 a$ \\
\hline & C. panzhihuaensis & $39.33 \pm 2.02 b$ & $37.07 \pm 0.81 c$ & $44.68 \pm 1.23 a^{*}$ & $40.04 \pm 1.75 b^{*}$ \\
\hline \multirow[t]{2}{*}{$P G$} & C. bifida & $34.73 \pm 0.78 a$ & $33.64 \pm 0.19 b$ & $33.77 \pm 0.07 b$ & $35.28 \pm 0.55 a$ \\
\hline & C. panzhihuaensis & $36.20 \pm 1.43 a$ & $35.06 \pm 0.58 a^{*}$ & $35.93 \pm 0.22 \mathrm{a}^{*}$ & $36.02 \pm 0.65 a$ \\
\hline \multirow[t]{2}{*}{$\mathrm{Pl}$} & C. bifida & $35.85 \pm 1.97 a b$ & $34.41 \pm 0.40 b$ & $34.96 \pm 0.63 a b$ & $36.51 \pm 0.25 a$ \\
\hline & C. panzhihuaensis & $35.21 \pm 0.83 b$ & $36.02 \pm 0.54 b^{*}$ & $38.27 \pm 0.47 a^{*}$ & $36.06 \pm 0.72 b$ \\
\hline \multirow[t]{2}{*}{ PS } & C. bifida & $33.11 \pm 0.02 b$ & $33.40 \pm 0.11 a$ & $33.40 \pm 0.18 a$ & $33.32 \pm 0.04 a$ \\
\hline & C. panzhihuaensis & $33.44 \pm 0.28 b$ & $33.82 \pm 0.15 a^{*}$ & $33.10 \pm 0.03 c^{*}$ & $33.29 \pm 0.25 b c$ \\
\hline \multirow[t]{2}{*}{ Phospholipids } & C. bifida & $36.04 \pm 1.83 a$ & $34.81 \pm 0.12 a$ & $35.03 \pm 0.22 a$ & $36.43 \pm 0.48 a$ \\
\hline & C. panzhihuaensis & $36.25 \pm 0.10 b$ & $36.03 \pm 0.24 b^{*}$ & $38.06 \pm 0.52 a^{*}$ & $36.49 \pm 0.47 b$ \\
\hline \multirow[t]{2}{*}{ MGMG } & C. bifida & $17.23 \pm 0.34 a$ & $17.41 \pm 0.42 \mathrm{a}$ & $17.50 \pm 0.22 \mathrm{a}$ & $17.15 \pm 0.08 a$ \\
\hline & C. panzhihuaensis & $17.41 \pm 0.12 b$ & $17.70 \pm 0.14 a$ & $17.49 \pm 0.12 \mathrm{ab}$ & $17.64 \pm 0.24 a$ \\
\hline \multirow[t]{2}{*}{ MGDG } & C. bifida & $35.22 \pm 0.53 a$ & $35.19 \pm 0.16 a$ & $35.30 \pm 0.07 a$ & $35.04 \pm 0.07 a$ \\
\hline & C. panzhihuaensis & $35.41 \pm 0.25 b$ & $35.26 \pm 0.05 b$ & $35.78 \pm 0.03 a^{*}$ & $35.66 \pm 0.07 a^{*}$ \\
\hline \multirow[t]{2}{*}{ DGDG } & C. bifida & $34.54 \pm 0.23 a$ & $34.43 \pm 0.24 a$ & $34.63 \pm 0.18 a$ & $33.57 \pm 0.46 b$ \\
\hline & C. panzhihuaensis & $34.71 \pm 0.07 c$ & $34.96 \pm 0.05 a^{*}$ & $34.87 \pm 0.02 b^{*}$ & $34.92 \pm 0.10 a^{*}$ \\
\hline \multirow[t]{2}{*}{ SQDG } & C. bifida & $39.68 \pm 0.36 a$ & $39.69 \pm 0.30 a$ & $39.61 \pm 0.04 a$ & $39.93 \pm 0.01 a$ \\
\hline & C. panzhihuaensis & $39.71 \pm 0.11 a$ & $39.77 \pm 0.11 a$ & $39.29 \pm 0.30 b$ & $39.76 \pm 0.06 a^{*}$ \\
\hline \multirow[t]{2}{*}{ Saccharolipids } & C. bifida & $37.39 \pm 0.29 a b$ & $36.94 \pm 0.57 b$ & $36.75 \pm 0.23 b$ & $37.52 \pm 0.17 a$ \\
\hline & C. panzhihuaensis & $36.91 \pm 0.42 a$ & $36.19 \pm 0.27 b^{*}$ & $37.20 \pm 0.38 a$ & $37.05 \pm 0.71 a$ \\
\hline \multirow[t]{2}{*}{ Total } & C. bifida & $36.79 \pm 0.75 a$ & $35.89 \pm 0.20 b$ & $35.99 \pm 0.11 b$ & $37.13 \pm 0.23 a$ \\
\hline & C. panzhihuaensis & $36.73 \pm 0.57 b$ & $36.15 \pm 0.22 b$ & $37.47 \pm 0.28 a^{*}$ & $36.89 \pm 0.64 a b$ \\
\hline
\end{tabular}

$\mathrm{ACL}=(\Sigma[\mathrm{n} \times \mathrm{mol} \%$ lipid $]) / 100$, where $\mathrm{n}$ is the number of acyl carbons in each lipid molecule. Different letters in the same row indicate significant differences between treatments within species and * indicates significant difference between species within treatment $(P<0.05)$. Data are mean \pm standard deviation $(n=5)$ $D G D G$ Digalactosyldiacylglycerol, MGDG Monogalactosyldiacylglycerol, MGMG monogalactosylmonoacylglycerol, $P A$ Phosphatidic acid, $P C$ Phosphatidylcholine, $P E$ Phosphatidylethanolamine, PG Phosphatidylglycerol, PI Phosphatidylinositol, PS Phosphatidylserine, SQDG Sulphoquinovosyldiacylglycerol

treatments but that of PS was lower following freezing. The ACL of total glycerophospholipids of C. panzhihuaensis was significantly higher than that of $C$. bifida after low-temperature treatments (Table 4).

Except for decreasing significantly in DGDG after recovery, the ACL of other classes of saccharolipids in $C$. bifida did not vary among the treatments (Table 4). The ACL of total saccharolipids of $C$. bifida showed no significant change after various treatments. For C. panzhihauensis, the ACL of MGMG, MGDG and DGDG increased significantly after cold or/and freezing treatment and that of SQDG decreased significantly after freezing treatment (Table 4). Except for SQDG, the ACL of all the classes of saccharolipids increased significantly after $3 \mathrm{~d}$ of recovery, compared to that of the control.
The ACL of total saccharolipids in C. panzhihauensis showed a significant decrease after cold treatment, which was significantly lower than that of C. bifida. The ACL of the total main membrane lipids (glycerophospholipids and saccharolipids) decreased significantly after lowtemperature treatments in C.bifida and increased significantly after freezing treatment in C. panzhihuaensis, being significantly higher than that of C. bifida.

Except for increasing significantly in PS after lowtemperature treatments, DBI of every glycerophospholipid class and total glycerophospholipids in C. bifida did not change (Table 5). For C. panzhihauensis, DBI of all the glycerophospholipid classes increased significantly after cold treatment, and those of PG and PI also increased significantly after freezing and recovery 
Table 5 Double bond index (DBI) of each main membrane lipid class in C. bifida and C. panzhihuaensis treated at low temperatures

\begin{tabular}{|c|c|c|c|c|c|}
\hline \multirow[t]{3}{*}{ Lipid class } & \multirow[t]{3}{*}{ Species } & \multicolumn{4}{|l|}{ Treatment } \\
\hline & & Control & Cold & Freezing & Recovery \\
\hline & & \multicolumn{4}{|l|}{$\mathrm{DBI}$} \\
\hline \multirow[t]{2}{*}{ PA } & C. bifida & $1.57 \pm 0.40 a$ & $1.48 \pm 0.09 a$ & $1.53 \pm 0.05 a$ & $1.60 \pm 0.23 a$ \\
\hline & C. panzhihuaensis & $1.87 \pm 0.12 b$ & $2.02 \pm 0.13 a^{*}$ & $1.81 \pm 0.07 b^{*}$ & $1.95 \pm 0.11 a b^{*}$ \\
\hline \multirow[t]{2}{*}{ PC } & C. bifida & $2.03 \pm 0.30 \mathrm{a}$ & $2.26 \pm 0.32 a$ & $2.19 \pm 0.21 \mathrm{a}$ & $2.70 \pm 0.35 a$ \\
\hline & C. panzhihuaensis & $2.84 \pm 0.37 b^{*}$ & $3.69 \pm 0.14 a^{*}$ & $2.43 \pm 0.16 b$ & $2.55 \pm 0.49 b$ \\
\hline \multirow[t]{2}{*}{ PE } & C. bifida & $2.43 \pm 0.38 a$ & $2.40 \pm 0.22 a$ & $2.34 \pm 0.08 a$ & $2.57 \pm 0.09 a$ \\
\hline & C. panzhihuaensis & $3.08 \pm 0.23 b^{*}$ & $3.53 \pm 0.22 a^{*}$ & $2.92 \pm 0.10 \mathrm{~b}^{*}$ & $2.98 \pm 0.29 b^{*}$ \\
\hline \multirow[t]{2}{*}{$P G$} & C. bifida & $1.74 \pm 0.23 a$ & $1.75 \pm 0.19 a$ & $1.82 \pm 0.10 \mathrm{a}$ & $1.71 \pm 0.06 a$ \\
\hline & C. panzhihuaensis & $1.69 \pm 0.18 c$ & $1.97 \pm 0.09 a b$ & $2.02 \pm 0.03 a^{*}$ & $1.85 \pm 0.06 b^{*}$ \\
\hline \multirow[t]{2}{*}{$\mathrm{Pl}$} & C. bifida & $1.65 \pm 0.33 a$ & $1.51 \pm 0.08 a$ & $1.59 \pm 0.06 a$ & $1.48 \pm 0.06 a$ \\
\hline & C. panzhihuaensis & $1.94 \pm 0.08 b$ & $2.05 \pm 0.08 a^{*}$ & $2.09 \pm 0.06 a^{*}$ & $2.06 \pm 0.04 a^{*}$ \\
\hline \multirow[t]{2}{*}{ PS } & C. bifida & $0.10 \pm 0.02 b$ & $0.37 \pm 0.13 a$ & $0.36 \pm 0.16 a$ & $0.29 \pm 0.04 a b$ \\
\hline & C. panzhihuaensis & $0.39 \pm 0.25 b$ & $0.80 \pm 0.18 a^{*}$ & $0.10 \pm 0.04 c^{*}$ & $0.25 \pm 0.20 b c$ \\
\hline \multirow[t]{2}{*}{ Phospholipids } & C. bifida & $1.70 \pm 0.35 a$ & $1.67 \pm 0.12 a$ & $1.72 \pm 0.10 a$ & $1.80 \pm 0.06 a$ \\
\hline & C. panzhihuaensis & $2.10 \pm 0.19 b$ & $2.50 \pm 0.14 a^{*}$ & $2.06 \pm 0.05 b^{*}$ & $2.13 \pm 0.20 b^{*}$ \\
\hline \multirow[t]{2}{*}{ MGMG } & C. bifida & $1.58 \pm 0.49 a$ & $1.92 \pm 0.67 a$ & $2.03 \pm 0.39 a$ & $1.44 \pm 0.13 a$ \\
\hline & C. panzhihuaensis & $1.93 \pm 0.18 b$ & $2.44 \pm 0.22 a$ & $2.02 \pm 0.23 b$ & $2.29 \pm 0.40 a b^{*}$ \\
\hline \multirow[t]{2}{*}{ MGDG } & C. bifida & $4.53 \pm 1.07 a$ & $4.70 \pm 0.41 a$ & $4.80 \pm 0.26 a$ & $4.08 \pm 0.14 a$ \\
\hline & C. panzhihuaensis & $5.27 \pm 0.13 c$ & $5.46 \pm 0.08 b^{*}$ & $5.77 \pm 0.08 a^{*}$ & $5.51 \pm 0.13 b^{*}$ \\
\hline \multirow[t]{2}{*}{ DGDG } & C. bifida & $2.93 \pm 0.44 b$ & $2.82 \pm 0.26 b$ & $3.11 \pm 0.33 b$ & $3.69 \pm 0.12 a$ \\
\hline & C. panzhihuaensis & $3.35 \pm 0.18 c$ & $3.88 \pm 0.18 a^{*}$ & $3.49 \pm 0.06 b c^{*}$ & $3.56 \pm 0.13 b$ \\
\hline \multirow[t]{2}{*}{ SQDG } & C. bifida & $6.96 \pm 0.03 a$ & $6.95 \pm 0.03 a$ & $6.95 \pm 0.01 a$ & $6.97 \pm 0.00 a$ \\
\hline & C. panzhihuaensis & $6.89 \pm 0.09 a$ & $6.95 \pm 0.03 a$ & $6.94 \pm 0.01 a$ & $6.95 \pm 0.01 a^{*}$ \\
\hline \multirow[t]{2}{*}{ Saccharolipids } & C. bifida & $5.56 \pm 0.34 a b$ & $5.32 \pm 0.24 b$ & $5.22 \pm 0.06 b$ & $5.75 \pm 0.09 a$ \\
\hline & C. panzhihuaensis & $5.55 \pm 0.12 a$ & $5.51 \pm 0.02 a$ & $5.84 \pm 0.17 a^{*}$ & $5.70 \pm 0.36 a$ \\
\hline \multirow[t]{2}{*}{ Total } & C. bifida & $3.99 \pm 0.46 a b$ & $3.55 \pm 0.16 b$ & $3.69 \pm 0.30 b$ & $4.39 \pm 0.17 a$ \\
\hline & C. panzhihuaensis & $4.55 \pm 0.07 b$ & $4.79 \pm 0.09 a^{*}$ & $4.59 \pm 0.06 b^{*}$ & $4.72 \pm 0.20 a^{*}$ \\
\hline
\end{tabular}

$\mathrm{DBI}=\Sigma(\mathrm{N} \times \mathrm{mol} \%$ lipid $) / 100$, where $\mathrm{N}$ is the number of double bonds in each lipid molecule. Different letters in the same row indicate significant differences between treatments within species and * indicates significant difference between species within treatment $(P<0.05)$. Data are mean \pm standard deviation $(n=5)$ $D G D G$ Digalactosyldiacylglycerol, MGDG Monogalactosyldiacylglycerol, MGMG monogalactosylmonoacylglycerol, $P A$ Phosphatidic acid, $P C$ Phosphatidylcholine, $P E$ Phosphatidylethanolamine, PG Phosphatidylglycerol, PI Phosphatidylinositol, PS Phosphatidylserine, SQDG Sulphoquinovosyldiacylglycerol

treatments, but the unsaturation level of PS decreased significantly after freezing treatment. The DBI of total glycerophospholipids in C. panzhihauensis increased significantly after cold treatment. Compared to those of $C$. bifida, the DBI of all the glycerophospholipid classes except PG of C. panzhihuaensis was significantly higher in cold-treated seedlings, which of PA, PE, PG and PI were significantly higher in freezing-treated and recovered seedlings (Table 5). The DBI and DBI/ACL of total glycerophospholipids of $C$. panzhihuaensis were significantly higher than those of C. bifida after various treatments (Table 5; Table S4).

Except that DBI of DGDG increased significantly after recovery, the DBI of every class of saccharolipids and the total saccharolipids in C. bifida were not affected by low temperatures. For C. panzhihuaensis, the DBI of MGDG in low-temperature-treated seedlings and those of MGMG and DGDG in cold-treated seedlings increased significantly compared to the control. Compared to those of $C$. bifida, the DBI of MGDG and DGDG in lowtemperature-treated seedlings of C. panzhihuaensis and that of total saccharolipids in freezing-treated seedlings were significantly higher. The DBI and DBI/ACL of total main membrane lipids were significantly higher in $C$. panzhihuaensis than those in C. bifida following low temperature and recovery treatments (Table 5; Table S4).

\section{Discussion}

Low temperature is a key factor limiting the introduction of tropical and subtropical plants, including cycads, 
to higher latitudes and altitudes. We previously showed that the freezing tolerance of $C$. panzhihuaensis was greater than that of C. bifida [23]. However, a systematic and in-depth study of the adaptation of the two species to low temperatures is still lacking. Lipids play key roles in diverse cellular processes and lipid metabolism is closely related to freezing tolerance of some plants [3, 24]. However, how lipids adjust under low temperature to regulate the tolerance of C. panzhihuaensis and $C$. bifida to low-temperature stress is not understood. Photosynthesis is a highly temperature-sensitive process [8]. Therefore, it is often used to reflect the adaptability of plants to temperature change. In the present study, the chlorophyll fluorescence parameters and lipid profiles of the two species subjected to cold, freezing and subsequent recovery were characterized.

\section{The reduction and loss of photosynthetic activities}

Chlorophyll fluorescence can sensitively reflect the physiological status of plants. The significant decrease of $\mathrm{Fv} / \mathrm{Fm}, \mathrm{Y}(\mathrm{II})$ and $\mathrm{rETR}$ and significant increase of $\mathrm{Y}(\mathrm{NO})$ with the decrease of temperatures in C. bifida demonstrate that photosynthetic activities of this species were affected, particularly severely by the freezing treatment. $\mathrm{Fv} / \mathrm{Fm}, \mathrm{Y}(\mathrm{II})$ and $\mathrm{rETR}$ reached to zero after $3 \mathrm{~d}$ of recovery and indicated that the photosynthetic apparatus of C. bifida was severely damaged and unable to recover. For C. panzhihuaensis, only Fv/Fm of the cold-treated seedlings decreased by $3.85 \%$ and $\mathrm{Fv} / \mathrm{Fm}, \mathrm{Y}(\mathrm{II})$ and rETR of the recovered seedlings decreased only by $3.97,17.63$ and $19.25 \%$, respectively, compared to those of the control. These values show that C. panzhihuaensis was relatively little affected by cold and freezing temperatures in comparison with C. bifida. The damaging effects of freezing on plant morphology might not appear immediately after treatments. They can, however, be more obvious after a period of recovery. We observed that freezing-treated leaves of $C$. bifida gradually became yellow and dry but those of C. panzhihuaensis remained green after $10 \mathrm{~d}$ of recovery. These results confirm that C. bifida is more sensitive to low temperatures than $C$. panzhihuaensis.

\section{The changes in composition of the lipid categories}

The metabolism of neutral glycerolipids is affected by low temperature and is related to plant tolerance to low temperatures [24]. For example, DAG and TAG accumulated and the DAG/TAG ratio decreased under freezing in Arabidopsis [16, 24]. The accumulation of TAG due to the conversion of DAG can contribute to the freezing tolerance of plants $[3,24]$. However, in this study the neutral glycerolipid content remained unchanged, and the DAG-TAG ratios of the treated seedlings were not significantly different from the control for either species (Fig. 1; Table S5). These results show that the freezing sensitivity of the two species had little relation to the neutral glycerolipid metabolism under freezing conditions. Glycerophospholipids and saccharolipids are the main extraplastidic and plastidic membrane lipids, respectively, in plants. Some data indicate that the two categories of lipids can degrade under low temperatures $[15,25]$. However, the glycerophospholipids and saccharolipids contents increased significantly after cold and freezing treatments in C. bifida and saccharolipids increased after cold treatment in C. panzhihuaensis. The sources of these accumulated lipids under low temperatures are not clear. The metabolic pathways of carbohydrates and lipids undergo cross talk to regulate energy homeostasis [26], but whether the increased lipids are due to the conversion of the stored carbohydrates requires clarification. For all of the treatments, the saccharolipids/glycerophospholipids ratio of $C$. panzhihuaensis was always significantly higher than that of C. bifida (Table S2). It is unclear if this is related to the higher freezing tolerance of C. panzhihuaensis.

Except that the saccharolipid and fatty acyl content increased significantly following cold and freezing treatments, respectively, the absolute contents of all the lipid categories of C. panzhihuaensis did not change after the various treatments (Fig. 1). For C. bifida, the absolute contents of all the lipid categories except neutral glycerolipids and sphingolipids varied with the treatments to different extent (Fig. 1). These results demonstrate that the lipid metabolism of $C$. bifida was more affected by cold and freezing treatments than $C$. panzhihuaensis. The proportion of some lipid categories changed after low-temperature treatments in both C. bifida and $C$. panzhihuaensis (Table 2). However, the proportions of all the lipid categories recovered to the original level after $3 \mathrm{~d}$ of recovery for C. panzhihuaensis, which of most lipid categories changed significantly for $C$. bifida in comparison with the control. This showed the plastic adjustment of lipid metabolism in C. panzhihauensis, which might be related to the greater tolerance of the species to low temperature. Phospholipids are major structural components of cell membranes and are involved in signal transduction and energy storage [27]. Prenol lipids (coenzyme Q here) are essential for energy metabolism in the electron transport system and also function as antioxidants within membrane systems [28]. The disorders of lipid metabolism after recovery such as the degradation of phospholipids and prenol lipids might contribute to the ultimate death of aboveground parts of C. bifida seedlings.

Lysophospholipids, sphingolipids and sterols are structural components of membranes and also important signaling molecules involved in plant development and environmental responses [29-31]. The accumulation of 
lysophospholipids and sphingolipids under stress might be detrimental to the cells [32, 33]. Besides sterol contents, the contents of lysophospholipids and sphingolipids of C. bifida were significantly higher than those of C. panzhihuaensis. Whether the differential tolerance of the two species to freezing is related to their different contents and change patterns of these lipids needs further study. Cuticular waxes are the primary structures of the cuticle and play crucial roles in plant defense against biotic and abiotic stress including drought and frost [34]. The significantly higher content of wax esters of $C$. panzhihuaensis following cold and freezing treatments might contribute to its higher freezing tolerance than $C$. bifida.

\section{Changes in composition of the main lipid classes and lipid species of glycerophospholipids and saccharolipids} PA can form nonbilayer lipid structures with MGDG or DAG during low temperature, disrupting the integrity of cell membrane $[35,36]$. Some studies showed that PA content increased dramatically under stresses such as freezing $[15,35]$. PA content remained unchanged in $C$. panzhihuaensis after various treatments but increased by 144.59 and $71.62 \%$ in C. bifida after cold and freezing treatments, respectively (Table 3 ). The maintenance of PA content in C. panzhihuaensis was conducive to keep the membrane stability, but the increase of PA in $C$. bifida after low-temperature treatments might pose a potential threat to membrane integrity. The significantly lower level of PA after cold and freezing treatments might confer higher freezing tolerance to $C$. panzhihuaensis.

The glycerophospholipid composition of various plant species presented different responses to stresses [11, 15, 37]. For C. bifida, the content of each glycerophospholipid class increased significantly after cold or/and freezing treatment but the PE, PS and total glycerophospholipid contents decreased significantly after $3 \mathrm{~d}$ of recovery (Fig. 1; Table 3). These results suggest that glycerophospholipid metabolism of C. bifida was dramatically affected by low temperature and the membrane was severely damaged following freezing treatment. For C. panzhihuaensis, different classes of glycerophospholipids showed different change trends following low-temperatute treatments. However, all the classes except for PG recovered to the original level. This demonstrated that PG was more sensitive to freezing temperature than other phospholipids in C. panzhihuaensis. Other studies have suggested that the level of high-melting-point PG molecules such as 32:0 and 32:1 is related to the sensitivity of plants to low temperatures $[38,39]$. The high-melting-point PG molecules were much lower in C. panzhihuaensis and showed a decrease and an increase after low temperature for $C$. panzhihuaensis and C. bifida, respectively (Fig. 2). This might be a factor involved in the freezing tolerance difference between the two species.

Saccharolipids are main lipids of the chloroplast envelope and thylakoid membrane, which play key roles in the photosynthetic process [40]. These lipids are likely to be degraded under some stresses [11, 41]. However, all the classes of saccharolipids in C. bifida increased significantly after low temperature treatments and all with the exception of DGDG and SQDG recovered to the control level after recovery (Table 3). The increase of saccharolipids after low temperatures suggests that seedlings of C. bifida positively resist the adverse effects of cold and freezing stresses on photosynthetic apparatus by stabilizing plastidic membranes. High DGDG/MGDG can be more conducive to maintaining bilayer membrane structure $[16,40]$. The substantial degradation of DGDG during post-freezing recovery indicates that the membrane integrity of $C$. bifida was damaged following freezing temperature. This is consistent with the results of chlorophyll fluorescence parameters, which showed the severe loss of photosynthetic activity of $C$. bifida. In contrast, the saccharolipids of C. panzhihuaensis including MGMG, MGDG and DGDG only responded to cold treatment. This might be an adaptive mechanism of $C$. panzhihuaensis to cold temperature, but the relevance of these findings with the freezing tolerance of the species requires further study. MGDG and DGDG are the main components of saccharolipids in most plants [10, 42]. However, the SQDG contents of C. bifida and C. panzhihuaensis accounted for 58.21 and $44.69 \%$ of the total saccharolipids, respectively, surpassing the MGDG and DGDG contents (Fig. 1; Table 3). A Similar phenomenon was found in some algae and lichens [43, 44]. SQDG was also found in cyanobacteria and nonphotosynthetic bacteria [43]. The high content of SQDG in the two Cycas species might be related to the highly diverse endophytic microbiome such as cyanobacteria and actinomycetes [45, 46]. It might also result from the adaptation of these species to nutrient-poor environments as P-starvation could promote SQDG accumulation [47].

\section{The changes in ACL and DBI of glycerophospholipids and saccharolipids}

ACL and DBI are important determinants of membrane fluidity, which are related to the development and environmental adaptability of plants [15]. The decrease of $\mathrm{ACL}$ and increase of DBI under low temperature enable the membranes to be more fluid, which contributes to plant tolerance to low temperatures [15]. The ACL and DBI of different classes of glycerophospholipids and saccharolipids showed different change trends following various treatments for each species. Among the 
glycerophospholipids, PG is the major component in chloroplast membranes. ACL of PG in C. bifida decreased significantly and DBI of PG in C. panzhihuaensis increased significantly after low-temperature treatments. This suggests that the two species adopt different strategies to increase the membrane fluidity. Our previous study suggested that ACL of PS was related to the plant lifespan, which can be accelerated by senescence but ceased to increase in plants near death [48]. In the present study, ACL of PS increased significantly after low temperatures and did not return to the control level after $3 \mathrm{~d}$ of recovery in C. bifida. ACL of PS varied with the decrease of temperature but recovered to the control level subsequently in C. panzhihuaensis. This implies that freezing-treated seedlings of $C$. bifida might gradually lose viability, while those of $C$. panzhihuaensis were not severely damaged.

In general, the ACL and DBI changes occurred in more lipid classes immediately after low temperatures in C. panzhihuaensis than those in C. bifida (Tables 4 and 5). The ACL and DBI of glycerophospholipids and saccharolipids remained unchanged in C. bifida. However, ACL and DBI of glycerophospholipids and ACL of saccharolipids responded to cold or freezing treatment, and they all recovered to the original level subsequently in $C$. panzhihuaensis (Tables 4 and 5). These results show that C. panzhihuaensis can better adjust the membrane fluidity to respond to the decreasing temperature. Based on the higher level of DBI/ACL (Table S4), the higher level of DBI of total glycerophospholipids could maintain a higher fluidity of extraplastidic membranes under low temperature in C. panzhihuaensis, although the ACL was significantly higher. For total saccharolipids, the ACL of C. panzhihuaensis was shorter after cold temperature and DBI was higher after freezing temperature compared to those of C. bifida. This could enable the seedlings of $C$. panzhihauensis to obtain more fluidity of plastidic membranes which are apt to be damaged under low temperature. The results were consistent with the chlorophyll fluorescence parameters that photosynthetic activity following treatments severely was lost in C. bifida but little changed in C. panzhihuaensis. The DBI of the total main membrane lipids of C. panzhihuaensis was significantly higher than that of C. bifida following all the treatments. The higher level of DBI after low temperature treatments might contribute to the higher freezing tolerance of C. panzhihuaensis.

\section{Conclusions}

The photosynthetic activity of C. bifida was more severely affected by low temperature than that of $C$. panzhihuaensis. The differential effects of freezing temperature were more obvious after $3 \mathrm{~d}$ of recovery. Seedlings of $C$. bifida lost almost all photosynthetic capacity, but $C$. panzhihuaensis seedlings were little affected. The results confirm previous work demonstrating that the freezing tolerance of $C$. panzhihuaensis was higher than that of $C$. bifida. The lipid composition of $C$. bifida was also more affected by cold and freezing treatments than $C$. panzhihuaensis. The proportions of all the lipid categories recovered to the original level for $C$. panzhihuaensis, but those of most lipid categories were changed significantly in C. bifida after $3 \mathrm{~d}$ of recovery. The homeostasis and plastic adjustment of lipid metabolism in C. panzhihauensis might be related to the greater tolerance of the species to low temperature than C. bifida. However, the severe degradation of glycerophospholipids and prenol lipids might be an important determinant of seedling death during the recovery period for C. bifida. The changes of ACL and DBI occurred in more lipid classes immediately after low temperatures in C. panzhihuaensis than those in C. bifida. The higher level of DBI of the main membrane lipids following low-temperature treatments might contribute to the higher freezing tolerance of $C$. panzhihuaensis. Lipid metabolism underwent different changes in seedlings of C. panzhihuaensis and C. bifida, which might be involved in the differential tolerance of the two species to low temperatures.

\section{Methods}

\section{Plant materials and treatments}

Seeds of C. panzhihuaensis were collected in Panzhihua. This was approved by the Administration Bureau of Panzhihua Cycas National Nature Reserve, Sichuan province. Seeds of C. bifida were collected from horticultural sources in Gejiu, Yunnan province. Seed collection was permitted by private land owners. The germinated four-year-old seedlings of $C$. bifida and $C$. panzhihuaensis were grown in a greenhouse in Southwest Forestry University and used to conduct the experiments. The seedlings of the two species were identified based on morphological characteristics by Shuangzhi Li, a taxonomist expert at Southwest Forestry University. Voucher specimens of C. panzhihuaensis (No. ZYL-001) and C. bifida (No. ZYL-002) were prepared and deposited in the Southwest Forestry University. The average temperature of the greenhouse was about $30 \pm 1^{\circ} \mathrm{C}$, and the daytime photosynthetic photon flux density was about $250-300 \mu \mathrm{mol} \mathrm{m}^{-2} \mathrm{~s}^{-1}$. All of the seedlings were planted in plastic pots containing humus and laterite soil $(1: 1 \mathrm{v} / \mathrm{v})$. For cold treatment, seedlings were transferred to artificial chambers at a constant temperature of $4{ }^{\circ} \mathrm{C}$ for $3 \mathrm{~d}$. The freezing and thaw treatments were conducted as described by Zhang et al. [49] and Arisz et al. [3] with some modifications. For the freezing treatment, the cold-treated seedlings were put in a programmable temperature incubator set at $0{ }^{\circ} \mathrm{C}$. Freezing was initiated 
by spraying ice cold water, and the temperature of the chamber was lowered at a rate of $2{ }^{\circ} \mathrm{Ch}^{-1}$ until $-5^{\circ} \mathrm{C}$ was reached. They were then maintained for $1.5 \mathrm{~h}$ at $5{ }^{\circ} \mathrm{C}$, after which the temperature was increased up to $4{ }^{\circ} \mathrm{C}$ at a rate of $2^{\circ} \mathrm{Ch}^{-1}$. The seedlings were thawed at $4{ }^{\circ} \mathrm{C}$ for $12 \mathrm{~h}$ and then recovered for $3 \mathrm{~d}$ at $30^{\circ} \mathrm{C}$. Seedlings, which were not subjected to cold and freezing treatments, served as the controls. There were 16 seedlings for each treatment.

\section{The chlorophyll fluorescence parameters}

Four seedlings were selected from each treatment, and one leaf was sampled from each seedling. Fluorescence parameters were tested at indoor temperature using a chlorophyll fluorometer (PAM-2500, Walz, Germany). Seedlings were dark-adapted for $30 \mathrm{~min}$ before measurements were conducted. The maximum quantum yield of photosystem II (PSII) (Fv/Fm), effective quantum yield of PS II (Y(II)), photochemical quenching coefficient (qP), non-photochemical quenching coefficient (NPQ), non-regulated $(\mathrm{Y}(\mathrm{NO}))$ and regulated $(\mathrm{Y}(\mathrm{NPQ}))$ nonphotochemical energy loss in PS II as well as relative electron transport rate (rETR) were measured.

\section{Sample preparation and lipid extraction}

Lipids were extracted according to MTBE method. Briefly, samples were first spiked with appropriate amount of internal lipid standards and then homogenized with $200 \mu \mathrm{L}$ water and $240 \mu \mathrm{L}$ methanol. After that, $800 \mu \mathrm{L}$ of MTBE was added and the mixture was ultrasound $20 \mathrm{~min}$ at $4{ }^{\circ} \mathrm{C}$ followed by sitting still for 30 $\mathrm{min}$ at room temperature. The solution was centrifuged at $14000 \mathrm{~g}$ for $15 \mathrm{~min}$ at $10{ }^{\circ} \mathrm{C}$ and the upper organic solvent layer was obtained and dried under nitrogen. Five seedlings were selected from each treatment, and one leaf was sampled from each seedling for lipid extraction.

\section{LC-MS/MS method for lipid analysis}

Reverse phase chromatography was selected for LC separation using CSH C18 column $(1.7 \mu \mathrm{m}, 2.1 \mathrm{~mm} \times 100$ $\mathrm{mm}$, Waters). The lipid extracts were re-dissolved in $200 \mu \mathrm{L} 90 \%$ isopropanol/ acetonitrile, centrifuged at $14000 \mathrm{~g}$ for $15 \mathrm{~min}$, finally $3 \mu \mathrm{L}$ of sample was injected. Solvent A was acetonitrile-water $(6: 4, \mathrm{v} / \mathrm{v})$ with $0.1 \%$ formic acid and $0.1 \mathrm{Mm}$ ammonium formate and solvent B was acetonitrile-isopropanol (1:9, v/v) with $0.1 \%$ formic acid and $0.1 \mathrm{Mm}$ ammonium formate. The initial mobile phase was $30 \%$ solvent B at a flow rate of $300 \mu \mathrm{L} / \mathrm{min}$. It was held for $2 \mathrm{~min}$, and then linearly increased to $100 \%$ solvent B in $23 \mathrm{~min}$, followed by equilibrating at $5 \%$ solvent $\mathrm{B}$ for $10 \mathrm{~min}$.

Mass spectra was acquired by Q-Exactive Plus in positive and negative mode, respectively. ESI parameters were optimized and preset for all measurements as follows: Source temperature, $300^{\circ} \mathrm{C}$; Capillary Temp, $350^{\circ} \mathrm{C}$, the ion spray voltage was set at $3000 \mathrm{~V}$, S-Lens RF Level was set at $50 \%$ and the scan range of the instruments was set at m/z 200-1800.

\section{Identification by lipid search}

"Lipid Search" is a search engine for the identification of lipid species based on MS/MS math. Lipid Search contains more than 30 lipid classes and more than 1,500,000 fragment ions in the database. Mass tolerance for precursor and fragment were both set to $5 \mathrm{ppm}$.

\section{Calculation of lipid double bond index (DBI) and acyl chain length $(A C L)$}

$\mathrm{ACL}=(\Sigma[\mathrm{n} \times \mathrm{mol} \%$ lipid $]) / 100$, where $\mathrm{n}$ was the number of acyl carbons in each lipid molecule; $\mathrm{DBI}=(\Sigma[\mathrm{N} \times \mathrm{mol}$ $\%$ lipid])/100, where $\mathrm{N}$ was the number of double bonds in each lipid molecule [15].

\section{Statistical analysis}

The data were subjected to one-way analysis of variance (ANOVA) with SPSS 15.0. Statistical significance was tested by Fisher's least significant difference (LSD) method. Comparisons between two species were evaluated by $\mathrm{T}$-test.

\section{Abbreviations}

ACL: Acyl chain length; CL: Cardiolipin; DAG: Diacylglycerol; DBI: Double bond index; DGDG: Digalactosyldiacylglycerol; Fv/Fm: The maximum quantum yield of photosystem II (PSII); MGDG: Monogalactosyldiacylglycerol; MGMG: monogalactosylmonoacylglycerol; NPQ: Non-photochemical quenching coefficient; PA: Phosphatidic acid; PC: Phosphatidylcholine; PE: Phosphatidylethanolamine; PG: Phosphatidylglycerol;

PI: Phosphatidylinositol; PIP: Phosphatidylinositol phosphate;

PS: Phosphatidylserine; QP: Photochemical quenching coefficient;

rETR: Relative electron transport rate; SQDG: Sulphoquinovosyldiacylglycerol; TAG: Triacylglycerol; Y(II): Effective quantum yield of PS II; Y(NO): Nonregulated non-photochemical energy loss in PS II; Y(NPQ): Regulated nonphotochemical energy loss in PS II

\section{Supplementary Information}

The online version contains supplementary material available at https://doi. org/10.1186/s12870-021-03158-4.

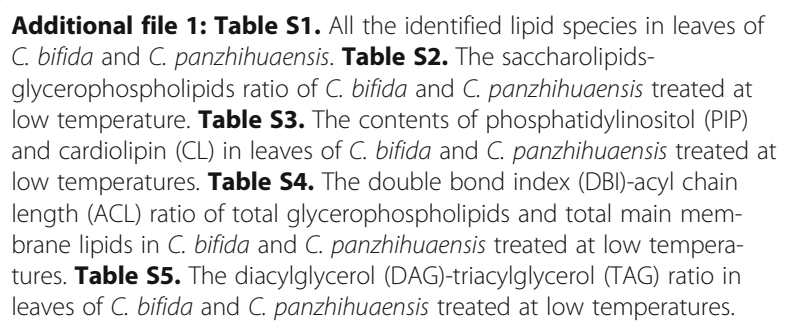

Additional file 1: Table S1. All the identified lipid species in leaves of C. bifida and C. panzhihuaensis. Table S2. The saccharolipidsglycerophospholipids ratio of C. bifida and C. panzhihuaensis treated at low temperature. Table S3. The contents of phosphatidylinositol (PIP) and cardiolipin (CL) in leaves of C. bifida and C. panzhihuaensis treated at low temperatures. Table S4. The double bond index (DBI)-acyl chain length $(\mathrm{ACL})$ ratio of total glycerophospholipids and total main membrane lipids in C. bifida and C. panzhihuaensis treated at low temperatures. Table S5. The diacylglycerol (DAG)-triacylglycerol (TAG) ratio in leaves of C. bifida and C. panzhihuaensis treated at low temperatures.

\section{Acknowledgements}

We acknowledge Shuangzhi Li for identification of C. panzhihuaensis and C bifida. 


\section{Authors' contributions}

$Y Z$, JW and $Z Y$ conceived and designed the research. $Y Z$ performed the experiments and wrote the manuscript. $Y Y, M W$ and $\mathrm{SH}$ provided assistance in data analysis. JW and ZY made helpful comments on the manuscript. All authors read and approved the final manuscript.

\section{Funding}

This work was supported by the National Natural Science Foundation of China $(32060094,31560093)$. The funding body did not play a role in the design of the study and collection, analysis, and interpretation of data and in writing the manuscript, but just provide the financial support.

\section{Availability of data and materials}

The datasets used and/or analyzed during the current study are available from the corresponding author on reasonable request.

\section{Declarations}

Ethics approval and consent to participate

Not applicable.

\section{Consent for publication}

Not applicable.

\section{Competing interests}

The authors declare that they have no competing interests.

\section{Received: 21 October 2020 Accepted: 4 August 2021}

Published online: 16 August 2021

\section{References}

1. Xin Z, Browse J. Cold comfort farm: the acclimation of plants to freezing temperatures. Plant Cell Environ. 2000;23(9):893-902. https://doi.org/10.104 6/j.1365-3040.2000.00611.x.

2. Shinkawa R, Morishita A, Amikura K, Machida R, Murakawa H, Kuchitsu K, et al. Abscisic acid induced freezing tolerance in chilling-sensitive suspension cultures and seedlings of rice. BMC Res Notes. 2013;6(1):351. https://doi.org/10.1186/1756-0500-6-351.

3. Arisz SA, Heo J-Y, Koevoets IT, Zhao T, van Egmond P, Meyer AJ, et al. DIACYLGLYCEROL ACYLTRANSFERASE1 contributes to freezing tolerance. Plant Physiol. 2018;177(4):1410-24. https://doi.org/10.1104/pp.18.00503.

4. Thomashow MF. Plant cold acclimation: freezing tolerance genes and regulatory mechanisms. Annu Rev Plant Physiol Plant Mol Biol. 1999;50(1): 571-99. https://doi.org/10.1146/annurev.arplant.50.1.571.

5. Kim JH, Kim JS, An BC, Chung BY. Alleviation of low-temperature photoinhibition in gamma-irradiated red pepper (Capsicum annuum L.) plants. J Plant Biol. 2006:49:353-7.

6. Foyer $\mathrm{CH}$, Harbinson J. Oxygen metabolism and theregulation of photosynthetic electron transport. In: Foyer $\mathrm{CH}$, Mullineaux PM, editors. Causes of photooxidative stress and amelioration of defense systems in plants. Boca Raton: CRC Press Inc; 1994. p. 1-42.

7. Anjum NA, Sofo A, Scopa A, Roychoudhury A, Gill SS, lqbal M, et al. Lipids and proteins-major targets of oxidative modifications in abiotic stressed plants. Environ Sci Pollut Res. 2015;22(6):4099-121. https://doi.org/10.1007/ s11356-014-3917-1.

8. Yamori W, Hikosaka K, Way DA. Temperature response of photosynthesis in $\mathrm{C}_{3}, \mathrm{C}_{4}$, and CAM plants: temperature acclimation and temperature adaptation. Photosynth Res. 2014;119(1-2):101-17. https://doi.org/10.1007/ s11120-013-9874-6.

9. Zhu JH, Dong $\mathrm{CH}$, Zhu JK. Interplay between cold-responsive gene regulation, metabolism and RNA processing during plant cold acclimation. Curr Opin Plant Biol. 2007:10(3):290-5. https://doi.org/10.1016/j.pbi.2007.04.010.

10. Liu M, Burgos A, Ma L, Zhang Q, Tang D, Ruan J. Lipidomics analysis unravels the effect of nitrogen fertilization on lipid metabolism in tea plant (Camellia sinensis L.). BMC Plant Biol. 2017;17(1):165. https://doi.org/10.1186/ s12870-017-1111-6.

11. Wang Y, Zhang X, Huang G, Feng F, Liu X, Guo R, et al. Dynamic changes in membrane lipid composition of leaves of winter wheat seedlings in response to PEG-induced water stress. BMC Plant Biol. 2020;20(1):84. https:// doi.org/10.1186/s12870-020-2257-1.
12. Hincha DK, Höfner R, Schwab KB, Heber U, Schmitt JM. Membrane rupture is the common cause of damage to chloroplast membranes in leaves injured by freezing or excessive wilting. Plant Physiol. 1987;83(2):251-3. https://doi.org/10.1104/pp.83.2.251.

13. Takahashi D, Li B, Nakayama T, Kawamura Y, Uemura M. Plant plasma membrane proteomics for improving cold tolerance. Front Plant Sci. 2013;4:90.

14. Kuch A, Warnecke DC, Fritz M, Wolter FP, Heinz E. Strategies for increasing tolerance against low temperature stress by genetic engineering of membrane lipids. In: Terzi M, Cella R, Falavigna A, editors. Current issues in plant molecular and cellular biology. Current plant science and biotechnology in agriculture. Dordrecht: Springer; 1995.

15. Zheng G, Li L, Li W. Glycerolipidome responses to freezing-and chillinginduced injuries: examples in Arabidopsis and rice. BMC Plant Biol. 2016; 16(1):70. https://doi.org/10.1186/s12870-016-0758-8.

16. Moellering ER, Muthan B, Benning C. Freezing tolerance in plants requires lipid remodeling at the outer chloroplast membrane. Science. 2010; 330(6001):226-8. https://doi.org/10.1126/science.1191803.

17. Fan J, Yan C, Roston R, Shanklin J, Xu C. Arabidopsis lipins, PDAT1 acyltransferase, and SDP1 triacylglycerol lipase synergistically direct fatty acids toward $\beta$-oxidation, thereby maintaining membrane lipid homeostasis. Plant Cell. 2014;26(10):4119-34. https://doi.org/10.1105/tpc.114.130377.

18. Durgud M, Gupta S, Ivanov I, Omidbakhshfard MA, Benina M, Alseekh S, et al. Molecular mechanisms preventing senescence in response to prolonged darkness in a desiccation-tolerant plant. Plant Physiol. 2018; 177(3):1319-38. https://doi.org/10.1104/pp.18.00055.

19. Hoffmann M, Hilton-Taylor C, Angulo A, Böhm M, Brooks TM, Butchart SH, et al. The impact of conservation on the status of the world's vertebrates. Science. 2010;330(6010):1503-9. https://doi.org/10.1126/science.1194442.

20. Cibrián-Jaramillo A, Marler TE, DeSalle R, Brenner ED. Development of ESTmicrosatellites from the cycad Cycasrumphii, and their use in the recently endangered Cycas micronesica. Conserv Genet. 2008;9(4):1051-4. https://doi. org/10.1007/s10592-007-9447-3.

21. Lindblad P, Costa JL. The cyanobacterial-cycad symbiosis. Biol Environ Proc Roy Irish Acad. 2002;102(1):31-3. https://doi.org/10.3318/BIOE.2002.102.1.31.

22. Xiao LQ, Ge XJ, Gong X, Hao G, Zheng SX. ISSR variation in the endemic and endangered plant Cycas guizhouensis (Cycadaceae). Ann Bot. 2004;94(1): 133-8. https://doi.org/10.1093/aob/mch119.

23. Xia Z, Ma H, Zheng Y. The physiological and biochemical responses of Cycas bifida and C panzhihuaensis to freezing stress. J Southwest Forestry Univ. 2020;40:1-8.

24. Tan W, Yang Y, Zhou Y, Huang L, Xu L, Chen Q, et al. DIACYLGLYCEROL ACYLTRANSFERASE and DIACYLGLYCEROL KINASE modulate triacylglycerol and phosphatidic acid production in the plant response to freezing stress. Plant Physiol. 2018;177(3):1303-18. https://doi.org/10.1104/pp.18.00402.

25. Yaneva IA, Vunkova-Radeva RV, Stefanov KL, Tsenov AS, Petrova TP, Petkov GO. Changes in lipid composition of winter wheat leaves under low temperature stress: effect of molybdenum supply. Biol Plant. 1995;37:561.

26. Yu L, Fan J, Yan C, Xu C. Starch deficiency enhances lipid biosynthesis and turnover in leaves. Plant Physiol. 2018;178(1):118-29. https://doi.org/10.1104/ pp.18.00539.

27. Wang X, Zhang W, Li W, Mishra G. Phospholipid signaling in plant response to drought and salt stress. In: Jenks MA, Hasegawa PM, Jain SM, editors. Advances in molecular breeding toward drought and salt tolerant crops. Dordrecht: Springer; 2007. p. 183-92. https://doi.org/10.1007/978-1-4020-5578-2_8.

28. Hayashi K, Ogiyama Y, Yokomi K, Nakagawa T, Kaino T, Kawamukai M. Functional conservation of coenzyme Q biosynthetic genes among yeasts, plants, and humans. PLoS One. 2014;9(6):e99038. https://doi.org/10.1371/ journal.pone.0099038.

29. Hou Q, Ufer G, Bartels D. Lipid signalling in plant responses to abiotic stress. Plant Cell Environ. 2016:39:1029-48.

30. Luttgeharm KD, Kimberlin AN, Cahoon EB. Plant sphingolipid metabolism and function. In: Nakamura Y, Li-Beisson Y, editors. Lipids in plant and algae development. Subcellular biochemistry, vol. 86. Cham: Springer; 2016. p. 249-86. https://doi.org/10.1007/978-3-319-25979-6_11.

31. Volkman JK. Sterols in microorganisms. Appl Microbiol Biotechnol. 2003; 60(5):495-506. https://doi.org/10.1007/s00253-002-1172-8.

32. Liu R, Jiang $W$, Yu D. Structure, metabolic pathway and function of sphingolipids in plants. Chin Bull Bot. 2009;44:619-28.

33. Wang D, Zheng G, Li W. Changes of membrane stability in potassiumstressed plants. Plant Diveristy Resour. 2014;36:595-602. 
34. Zhou L, Ni E, Yang J, Zhou H, Liang H, Li J, et al. Rice OsGL1-6 is involved in leaf cuticular wax accumulation and drought resistance. PLoS One. 2013; 8(5):e65139. https://doi.org/10.1371/journal.pone.0065139.

35. Welti R, Li W, Li M, Sang Y, Biesiada H, Zhou HE, et al. Profiling membrane lipids in plant stress responses: role of phospholipase $D$ alpha in freezinginduced lipid changes in Arabidopsis. J Biol Chem. 2002;277(35):31994-2002. https://doi.org/10.1074/jbc.M205375200.

36. Moellering ER, Benning C. Galactoglycerolipid metabolism under stress: a time for remodeling. Trends Plant Sci. 2011;16(2):98-107. https://doi.org/10.1 016/j.tplants.2010.11.004

37. Gasulla F, Vom Dorp K, Dombrink I, Zaehringer U, Gisch N, Doermann P, et al. The role of lipid metabolism in the acquisition of desiccation tolerance in Craterostigma plantagineum: a comparative approach. Plant J. 2013;75(5):726-41. https://doi.org/10.1111/tpj.12241.

38. Murata N, Ishizaki-Nishizawa O, Higashi S, Hayashi H, Tasaka Y, Nishida I. Genetically engineered alteration in the chilling sensitivity of plants. Nature. 1992;356(6371):710-3. https://doi.org/10.1038/356710a0.

39. Sakamoto A, Sulpice R, Hou CX, Kinoshita M, Higashi SI, Kanaseki T, et al. Genetic modification of the fatty acid unsaturation of phosphatidylglycerol in chloroplasts alters the sensitivity of tobacco plants to cold stress. Plant Cell Environ. 2004;27(1):99-105. https://doi.org/10.1046/j.0016-8025.2003.01131.x.

40. Dörmann P, Benning C. Galactolipids rule in seed plants. Trends Plant Sci. 2002; (3):112-8. https://doi.org/10.1016/S1360-1385(01)02216-6.

41. Zheng G, Li W. Profiling membrane glycerolipids during $\gamma$-ray-induced membrane injury. BMC Plant Biol. 2017;17(1):203. https://doi.org/10.1186/s12 870-017-1153-9.

42. Sakurai I, Shen JR, Leng J, Ohashi S, Kobayashi M, Wada H. Lipids in oxygenevolving photosystem II complexes of cyanobacteria and higher plants. J Biochem. 2006;140(2):201-9. https://doi.org/10.1093/jb/mvj141.

43. Harwood JL, Okanenko AA. Sulphoquinovosyl diacylglycerol (SQDG)-the sulpholipid of higher plants. In: Abrol YP, Ahmad A, editors. Sulphur in plants. Dordrecht: Springer; 2003. p. 189-219. https://doi.org/10.1007/97894-017-0289-8 11

44. Bychek-Guschina IA. Study of biochemical aspects of lichen symbiosis. 1 Lipids and fatty acids in cultured lichen symbionts. Biohimiya. 1997;62:57480 (in Russian).

45. Zheng Y, Gong X. Niche differentiation rather than biogeography shapes the diversity and composition of microbiome of Cycas panzhihuaensis. Microbiome. 2019;7(1):152. https://doi.org/10.1186/s40168-019-0770-y.

46. Zheng Y, Chiang T-Y, Huang C-L, Gong X. Highly diverse endophytes in roots of Cycas bifida. J Microbiol. 2018;56(5):337-45. https://doi.org/10.1007/ s12275-018-7438-3.

47. Kalisch B, Dörmann P, Hölzl G. DGDG and glycolipids in plants and algae. In: Nakamura Y, Li-Beisson Y, editors. Lipids in plant and algae development. Subcellular biochemistry, vol. 86. Cham: Springer; 2016. p. 51-83. https://doi. org/10.1007/978-3-319-25979-6_3.

48. Li Y, Zheng G, Jia Y, Yu X, Zhang X, Yu B, et al. Acyl chain length of phosphatidylserine is correlated with plant lifespan. PLoS One. 2014;9(7): e103227. https://doi.org/10.1371/journal.pone.0103227.

49. Zhang Z, Hu X, Zhang Y, Miao Z, Xie C, Meng X, et al. Opposing control by transcription factors MYB61 and MYB3 increases freezing tolerance by relieving c-repeat binding factor suppression. Plant Physiol. 2016;172(2): 1306-23. https://doi.org/10.1104/pp.16.00051.

\section{Publisher's Note}

Springer Nature remains neutral with regard to jurisdictional claims in published maps and institutional affiliations.

Ready to submit your research? Choose BMC and benefit from:
- fast, convenient online submission
- thorough peer review by experienced researchers in your field
- rapid publication on acceptance
- support for research data, including large and complex data types
- gold Open Access which fosters wider collaboration and increased citations
- maximum visibility for your research: over 100M website views per year
At BMC, research is always in progress.
Learn more biomedcentral.com/submissions

